\title{
La extensión y su vínculo con la docencia: Una visión integral
}

\section{The University Extension and Its Link With Teaching: A Comprehensive Vision}

\author{
Melvin Bermúdez Elizondo \\ Universidad Nacional \\ Sede Regional Brunca, Campus Pérez Zeledón \\ Pérez Zeledón, Costa Rica \\ melvin.bermudez.elizondo@una.cr \\ Carlos Ronald Borbón Sandi \\ Universidad Nacional \\ Sede Regional Brunca, Campus Pérez Zeledón \\ Pérez Zeledón, Costa Rica \\ rborbon@una.cr
}

Recibido: 22/02/2018 Aceptado: 07/06/2018

Resumen. La gran diferencia entre la educación superior privada y la pública, radica no solamente en su coste, sino más bien en la formación integral y humanista que caracteriza a la educación pública estatal, no obstante, este discurso parece desgastado para enfrentar las críticas de algunos miembros de la clase política de este país, que han visto en la educación pública un gasto en lugar de una inversión. Para la próxima década, la universidad debe proyectarse a la sociedad civil, en este sentido los proyectos de extensión universitaria han de ser la principal herramienta para lograr tal proyección. Pero, además la vinculación de la docencia y la extensión universitaria podrá retroalimentar los currículos de las carreras, fortaleciendo con ello la formación profesional de los estudiantes. En el pasado la extensión se concebía como un fin en sí mismo, con poca articulación con la docencia, puesto que los productos finales estaban orientados más hacia la sociedad civil que a nivel interno. La vinculación entre la extensión y la docencia es y debería ser un proceso de articulación en los proyectos 
ReVista Universidad en Diálogo • Vol. 8, N. ${ }^{\circ}$ 2, Julio-Diciembre, 2018, pp. 71-80

ISSN 2215-2849 • EISSN: 2215-4752

DOI: http://dx.doi.org/10.15359/udre.8-2.5

de extensión. La experiencia atesorada con el proyecto de extensión desarrollado en la Sede Regional Brunca en los años 2015-2016 ha contribuido para ejemplificar el vínculo entre estas dos áreas del quehacer sustantivo de la universidad.

Palabras claves: docencia, integración, articulación, experiencia.

Abstract. The significant difference between private and public higher education lies not only in its cost but rather in the comprehensive and humanistic formation that characterizes the state public education. However, this discourse seems worn out to face the criticism of some members of the political class of this country, who have seen in public education an expense instead of an investment. For the next decade, the university should be projected to civil society; in this sense, university extension projects should be the main tool to achieve such a projection. However, also, the link between teaching and university extension can provide feedback to the curricula of careers, strengthening thereby the professional training of the students. In the past, the university extension was conceived as an end in itself, with little articulation with teaching, since the final products were oriented more towards civil society than internally. The link between extension and teaching is and should be a process of articulation in extension projects. The experience treasured with an extension project developed in the Brunca Regional Campus, in 2015 2016, has contributed to exemplify the link between these two areas of the substantive work of the university.

Keywords: teaching, integration, articulation, experience.

\section{Introducción}

A pesar de que el tema de la integralidad en los proyectos de extensión ha sido muy discutido a nivel internacional, en la UNA parece haber estado en un periodo de reposo, puesto que a nivel conceptual siempre se ha subrayado la importancia de realizar proyectos integrados, pero en la práctica los mismos casi nunca se han logrado, sobre todo aquellos que deberían integrar a la docencia. Los/as investigadores/as y extensionistas, a pesar de ser también docentes, poco han logrado integrar los proyectos que desarrollan con la docencia. El sentido de integralidad habrá de ser abordado con mayor profundidad en esta nueva etapa.

La experiencia desarrollada con la ejecución del proyecto de extensión 269-14 ha servido de base para poner sobre la palestra la necesidad de integrar la extensión y la docencia, dado que en el mismo se logró hacer 
un esfuerzo para integrar estas dos actividades, además de hacerlo con dos áreas disciplinarias, la administración y la ingeniería en sistemas.

Este trabajo describe cómo se originó de forma espontánea la integración entre la extensión y la docencia durante la ejecución del proyecto de extensión, siendo esta integración una lección aprendida de gran relevancia para el futuro de la extensión en la Sede Regional Brunca.

Dentro de este contexto de desarrollo del proyecto, es necesario reconocer la activa y proactiva participación que tuvieron los actores del proyecto, desde el personal académico responsable del mismo hasta los y las estudiantes que, en el papel de estudiantes asistentes, colaboradores/as y participantes, desarrollaron actividades que lograron vincular la extensión con la docencia. Esta integración fue básica para que el currículo de las carreras de Administración e Ingeniería en Sistemas pasará de la teoría a la práctica, puesto que de forma conjunta los contenidos teóricos conceptuales fueron desarrollándose con la aplicación práctica de los mismos.

\section{Objetivos}

1. Describir la forma en la que los contenidos teóricos de algunos cursos de la carrera de Administración encontraron una aplicación práctica en un proyecto de extensión.

2. Concientizar al personal académico de las unidades académicas y sedes para que logren coordinar y articular los contenidos de los cursos de las carreras con la aplicación práctica en proyectos de extensión.

3. Incentivar que más académicos y académicas puedan formular proyectos de extensión y con ello articular la docencia y la extensión desde una perspectiva integral.

\section{Marco conceptual-referencial}

Para abordar el tema de proyectos integrados es necesario en primera instancia desarrollar el concepto de integralidad, pero visualizado desde la perspectiva de la investigación. En este sentido, en el trabajo desarrollado por Tommasino y Rodríguez (2010) -donde se refleja este proceso-se menciona: 
ReVista Universidad en Diálogo • Vol. 8, N. ${ }^{\circ}$ 2, Julio-Diciembre, 2018, pp. 71-80

ISSN 2215-2849 • EISSN: 2215-4752

DOI: http://dx.doi.org/10.15359/udre.8-2.5

...un primer movimiento realizado fue pasar de considerar la extensión como un aspecto importante en el proceso de Reforma Universitaria, a considerarla como el punto de partida para el desarrollo de las prácticas integrales, y en consecuencia pensarla como una de las herramientas de transformación de la Universidad. La función de la extensión no puede ser entendida como algo aislado, encapsulada en un gueto, sino que debe ponerse en interacción en el acto educativo y formar parte de manera naturalizada en el mismo. De ahí que se afirme que la curricularización de la extensión o la acreditación curricular de la extensión, no puede darse de una forma independiente y aislada, en un lugar específico de la formación de los estudiantes (p. 21).

Sugieren los autores un aspecto de vital importancia y es el hecho de que la extensión (al igual que la investigación) no debe ser concebida como una actividad independiente de la actividad académica, sino más bien como un complemento ideal para lograr vincular la universidad con la sociedad y la teoría con la práctica.

En este contexto, Boaventura de Souza Santos (2010) plantea que las reformas de la universidad en este nuevo siglo "deben partir del supuesto de que en el siglo XXI solo habrá universidad cuando haya formación de grado y posgrado, investigación y extensión. Sin cualquiera de éstas, habrá enseñanza superior pero no universidad" (p. 43). Plantea este autor la necesidad (no la posibilidad) de que la universidad desarrolle su acción sustantiva, no solo con la docencia, sino también con la extensión y la investigación.

En Costa Rica, la gran diferencia entre la educación superior privada y las universidades estatales radica precisamente en las actividades de investigación, extensión y producción que realizan estas universidades, pero a pesar de ello pareciera que las actividades se realizan con poca integralidad, enfocadas en sus propósitos y objetivos específicos, sin mirar el valor agregado que pueden producir en conjunción con la docencia.

Tomando como referencia la resolución del Consejo Directivo Central de la Universidad de la República emitido en octubre del 2009, los autores Tommasino y Rodríguez (2010) toman la definición de la extensión como: 
Proceso educativo transformador donde no hay roles estereotipados de educador y educando, donde todos puedan aprender y enseñar.

Proceso que contribuya a la producción de conocimiento nuevo que vincula críticamente el saber académico con el saber popular.

Proceso que tiende a promover formas asociativas y grupales que aporten a superar problemáticas significativas a nivel social.

Es una función que permite orientar líneas de investigación y planes de enseñanza, generando compromiso universitario con la sociedad y con la resolución de sus problemas.

En su dimensión pedagógica, constituye una metodología de aprendizaje integral y humanizadora (pp. 15-16).

Teniendo claro el concepto de integralidad y la relación entre extensión y docencia, se describirá a continuación el marco referencial sobre el cual se plantea la propuesta de vincular la extensión con la docencia desde una perspectiva integral.

El proyecto de extensión denominado Fortalecimiento de la Competitividad de Diez Microempresas del Cantón de Pérez Zeledón, Región Brunca, dentro de un concepto de acompañamiento a las organizaciones, pretendió aumentar las capacidades y competencias técnico-administrativas de las organizaciones seleccionadas, provenientes del ámbito asociativo y de iniciativas privadas ubicadas en este cantón.

Dos componentes resaltaron en el proyecto, el primero de ellos enfocado en la activa participación de estudiantes de las carreras de Administración e Ingeniería en Sistemas, tanto estudiantes asistentes del proyecto como estudiantes avanzados/as de ambas carreras, en donde los/as primeros/as se encargaron de la redacción (bajo supervisión) de los procedimientos y manuales de las áreas funcionales de las organizaciones y los/as segundos/ as realizaron trabajos prácticos, sobre todo en el desarrollo de sistemas de información administrativa para dichas organizaciones.

En los resultados concernientes a la vinculación de la extensión con la docencia se proporcionarán más elementos de juicio que evidencian cómo se dio esta integración. 
ReVista Universidad en Diálogo • Vol. 8, N. ${ }^{\circ}$ 2, Julio-Diciembre, 2018, pp. 71-80

ISSN 2215-2849 • EISSN: 2215-4752

DOI: http://dx.doi.org/10.15359/udre.8-2.5

\section{Marco metodológico}

La metodología que sigue el trabajo se basa en un análisis cualitativo, en donde la teoría aplicada en la vinculación de la teoría con la práctica se basa en aprender haciendo, este "debía ser un programa de enseñanza práctico, centrado en la experiencia de los estudiantes y que implicara a la vez un hacer y una prueba" (Schmidt, 2006).

El trabajo práctico "suministra magníficas oportunidades para aprender las materias de los programas de estudio, no solo como información, sino como un conocimiento adquirido a través de las situaciones de la vida" (Schmidt, 2006).

El tipo de diseño que más se adaptó a este proyecto fue el de investigaciónacción, debido a que es una forma de estudiar, de explorar, una situación social con la finalidad de mejorarla, en la que se implican como "indagadoras" las personas implicadas en la realidad investigada (Suárez, 2002). John Elliot (2005), uno de los autores más reconocidos en cuanto a este diseño de investigación, señala que la investigación-acción es el "estudio de una situación social con el fin de mejorar la calidad de la acción dentro de la misma".

En este tipo de diseño de investigación es necesario un alto compromiso de las personas participantes o sujetos de información para el proyecto, así como una gran motivación por obtener los resultados esperados.

\section{Resultados}

En relación con los resultados del proyecto, en lo que respecta a la integración entre la extensión y la docencia se puede afirmar que dicha integración se originó más de una forma coyuntural que como algo planificado dentro del proyecto.

La motivación de las personas responsables del proyecto en cuanto a llegar a los encargados y las encargadas de los cursos de tercer y cuarto año de las carreras de Administración e Ingeniería en Sistemas constituyó un punto de inflexión que coadyuvó no solo al éxito del proyecto en cuanto a los objetivos planteados, sino también en cuanto a que muchos cursos lograron integrar la teoría con la práctica.

A continuación, se enunciarán los principales procesos de integración que se dieron durante la ejecución del proyecto. 


\section{Carrera de Administración}

Con la activa participación de seis estudiantes asistentes de la carrera de Administración de tercer y cuarto nivel y al menos quince estudiantes en total, se lograron realizar las siguientes integraciones con el proyecto de extensión:

- En el curso Preparación y Evaluación de Proyectos se integró un grupo de estudiantes que realizaron su trabajo práctico del curso en la Asociación de Productores Lácteos de La Sierra de Platanares, proyecto que estableció un análisis de prefactibilidad para la instalación y operación de una planta agroindustrial para dicha asociación.

- En esta misma asociación, pero en el curso de Mercadeo, un grupo de estudiantes desarrolló un plan de mercadeo (exigido en el curso) con el planteamiento de medios de comercialización en forma digital, creación de página web y un perfil de Facebook para dar a conocer los productos.

- También en el curso de Gestión de Presupuesto un grupo de estudiantes logró desarrollar su trabajo práctico en una de las organizaciones participantes en el proyecto.

- La Asociación de Productores de Frutas de la Región Brunca (ASOFRUBRUNCA) fue otra de las organizaciones en donde se realizaron tres trabajos prácticos de cursos de la carrera de Administración, específicamente en los cursos de Mercadeo, Investigación de Mercados y Proyectos. Además, en esta organización se realizó un trabajo final de licenciatura por parte de dos estudiantes de la carrera de Administración.

\section{Carrera de Ingeniería en Sistemas}

En esta carrera se generó una integración profunda en los cursos de Ingeniería en Sistemas I, Ingeniería en Sistemas II e Ingeniería en Sistemas III, puesto que en estos cursos catorce de los diecisiete estudiantes, que en el 2015 cursaban su tercer año de carrera, se comprometieron con el proyecto y asumieron como trabajo práctico de estos cursos el desarrollar un sistema de información para ocho de las diez empresas participantes en el proyecto de extensión. 
Sin duda alguna, la participación de este grupo de estudiantes con sus proyectos de docencia, aplicados en un proyecto de extensión, generó el entusiasmo y dedicación que solo genera el compromiso de integrar los conocimientos teóricos y conceptuales con la aplicación práctica de los mismos.

\section{Discusión y conclusiones}

Dentro de las principales conclusiones y aportando a la discusión del Congreso, centrada en la formulación de políticas de extensión para los próximos diez años, se tiene que:

- La práctica común de muchas universidades y de muchos programas curriculares se basa en la docencia intramuros, con escaso contacto del estudiantado con el mundo real y con poca o nula interacción con proyectos de investigación y extensión.

- Se propone entonces poder llevar los programas curriculares de las carreras a la aplicación práctica en casos reales de la sociedad civil y que coadyuven en la realidad cotidiana que enfrentan empresas y organizaciones, de forma que la vinculación de la teoría con la práctica sea un eje transversal en el desarrollo de los currículos. Los proyectos de extensión pueden sin lugar a dudas ser una invaluable herramienta en este proceso.

- En la ejecución del proyecto de extensión universitaria en la Sede Regional Brunca existió una excelente coordinación entre el trabajo a desarrollar en las organizaciones y los contenidos teóricos de cursos, mismos que fueron aplicados a las organizaciones y empresas participantes en el proyecto FUNDER.

- De ahí que la reflexión, además de pretender una integración entre la docencia y la extensión, invita a iniciar con un proceso intenso de articulación y coordinación entre el personal académico de las distintas unidades y carreras, de forma que el proceso de integración sea construido desde el convencimiento y la motivación de mejorar la calidad de enseñanza que se le está ofreciendo a la comunidad estudiantil de pregrado y grado.

- El estudiantado mostró un alto grado de compromiso, motivación e interés cuando logró identificar la relación de la teoría y su aplicación práctica en condiciones reales. 
- Es por lo anterior que se deben buscar los métodos o las metodologías apropiadas para que sea la comunidad estudiantil la que coadyuve en los procesos de integralidad, es decir, lograr que el propio estudiantado sea el que solicite o, por qué no, exija al personal docente que exista mayor vinculación de la teoría con la práctica, ya que los tiempos han cambiado y los procesos de formación integral en competencias solo se logran si se avanza de una educación intramuros a una formación extramuros.

- La disposición de los y las docentes de los cursos ante la motivación de las personas responsables del proyecto de poder desarrollar los trabajos prácticos de sus cursos en las empresas del proyecto fue fundamental para lograr la integración señalada.

- De lo anterior se puede rescatar el hecho de que la motivación, y más aún la pasión, es lo que puede marcar la diferencia entre los resultados de un proyecto común y de un proyecto extraordinario. Pero qué motiva a los y las docentes, o más bien qué desmotiva al personal docente y le impide realizar un esfuerzo adicional o plantear proyectos de extensión e investigación, quedándose inmóvil en el aula, sin integrar la teoría con la práctica, pudiendo hacerlo. Encontrar los factores motivacionales y al mismo tiempo aquellos que causan desmotivación es parte de los retos de las políticas de extensión de la próxima década.

- El cumplimiento de la acción sustantiva de la universidad será fortalecido si se puede lograr una adecuada coordinación y articulación entre la extensión y la docencia. Situación que debe ser exigida desde los mismos requisitos de presentación de los proyectos, brindando un mayor porcentaje de calificación a los proyectos que evidencien de forma clara la forma en la que se podrá articular la extensión con la docencia: cuáles cursos podrán integrarse, cuántos trabajos prácticos podrán desarrollarse, cuántos estudiantes saldrán de las aulas para integrarse a la sociedad civil en los proyectos, son algunos ejemplos de indicadores de evaluación que pueden ser planteados.

- La sistematización, producción y difusión de los resultados de los proyectos de extensión deben reflejar que ha existido una articulación integral entre la extensión y la docencia. En este sentido, debe ser un requisito de los proyectos la presentación de 
los trabajos de docencia que se desarrollan en los mismos, como productos concretos, tanto en sus informes parciales como en el informe final de cierre.

- Los trabajos prácticos que realiza el estudiantado en un determinado curso, desarrollados en proyectos de extensión universitaria, deben ser visibilizados, difundidos y promocionados como una buena práctica de la docencia universitaria; para ello se pueden utilizar medios digitales, una revista de extensión más abierta a la difusión de los resultados y productos desarrollados por los y las estudiantes en cursos de carrera en donde han desarrollado trabajos prácticos, al final la vinculación del estudiantado con la sociedad civil es también una forma de hacer extensión.

\section{Referencias}

Boaventura de Souza, S. (2010). La universidad del siglo XXI. Para una reforma democrática y emancipatoria de la universidad. TrilceExtensión Universitaria. Montevideo.

Elliot, J. (2005). La investigación-acción en educación. España: Editorial Morata.

Suárez, M. (2002). Algunas reflexiones sobre la investigación-acción colaboradora en la educación. Revista Electrónica de Enseñanza de las Ciencias, 1(1).

Schmidt, S. (2006). El aprender haciendo viene desde John Dewey. Recuperado de http://www.minam.gob.pe/proyecolegios/Curso/ curso-virtual/Modulos/modulo2/3Secundaria/m2_secundaria/ Aprender_haciendo-John_Dewey.pdf

Tommasino, H. y Rodríguez, N. (2010). Tres tesis básicas sobre extensión y prácticas integrales en la Universidad de la República. En AA.VV., Cuadernos de Extensión $N^{o}$. 1. Integralidad: tensiones y perspectivas (pp. 19-42). Montevideo: CSEAM. 